\title{
A Cross-cultural Comparison of Medical Science Subject Classification in the KDC and the DDC
}

\author{
InKyung Choi \\ University of Wisconsin Milwaukee \\ ichoi@uwm.edu
}

\author{
Min Sook Park \\ University of Wisconsin Milwaukee \\ minsook@uwm.edu
}

\begin{abstract}
This study reports the preliminary results of a cross-cultural comparison of Medical Science in the Korean Decimal Classification (KDC) and in the Dewy Decimal Classification (DDC). Despite having similar purposes, to serve the public and emphasize the significance of standardization in medical science, a comparison of the two classification systems shows the influences of social and cultural inferences of classification systems in medicine.
\end{abstract}

\section{Keywords}

Classification, Cross-cultural comparison, Medical Science classification, KDC, DDC.

\section{INTRODUCTION}

This study compares subject of Medical Science in the Korean Decimal Classification (KDC) and that in the Dewy Decimal Classification (DDC). Although classification systems are necessary to provide a framework in which to scientifically study disease and conditions in an orderly fashion (Armitage, 1999), social and cultural influences are inevitable in the development of a classification system (Gartner, 2016). This cross-cultural comparison study investigates the sociocultural influences on Knowledge Organization Systems (KOSs) designed to organize medical information resources in two distinct sociocultural contexts.

Medical classification, in general, transforms description of medical diagnosis and procedures into universal medical code numbers. At the same time, the scheme should be able to meet the needs of various fields of knowledge (Ballard, 1921). Establishing a standard medical language and classification scheme is essential for ensuring uniformity as well as enhancing collection and access to medical data and resources (Chute, 2000). Medical classification consists of terminologies, which are categorized into nomenclatures systematic listings of the proper names and a medical classification system that organizes medical terminologies into categories (Ballard, 1921).

However, subject classification, like medical science, is not free from social and cultural influences. Classification is a human creation and bears the imprints of its progenitors in the form it takes (Gartner, 2016). Terminologies and classi-

$81^{\text {st }}$ Annual Meeting of the Association for Information Science \& Technology | Vancouver, Canada | Nov. 10 - 14, 2018 Author(s) Retain Copyright fication schemes are inevitably subjective and selective (Gartner, 2016). The $D D C$ and $K D C$ are not an exception. The $K D C$, the widely used scheme used in the majority of libraries and publication operations in Korea, was developed based on the $D D C$, but modified to meet the needs in Korea. Besides, although both of classification systems have been developed and deployed mainly to enhance the general public's access to information resources, they are essentially designed to serve different societies, and reflect their socio-cultural differences. This preliminary study reports the results of a comparison of the two classification systems, with a focus on medical science along with anecdotal findings. As a preceding state of the comparison, the analysis focused on semantic and structural differences in the $D D C$ and the $K D C$.

\section{METHOD}

This study used the cross-cultural analysis method, which refers to a comparison of various sociological or cultural factors to assess the similarities and diversities occurring in two or more cultures or societies (U.S. National Library of Medicine, 2018).

The researchers of this study manually compared the most recent editions of the $K D C$ and the $D D C$ to examine differences in the medical science subject. The sixth edition of $K D C^{\text {'s }} 510$ to 519 on the subject of medical science and the twenty-third edition of the $D D C$ 's 610 to 619 on the subject of medicine and health were used. Particular considerations were given to two classificatory features: semantic content and structure. These two features are constructs of classification in which the social and cultural influences are evident. The semantic contents of classification, such as terminology, synonyms, and antonyms, can represent the interests of a certain group (Olson, 2010). These linguistic entities serves as a framework for organizing, analyzing, and interpreting actions, motives, attitudes, and values (Hantrais, 2008), depending on its political, cultural, and moral context. The structure of the classification, such as hierarchy, results from the cultural and intellectual infrastructure (Olson, 2010). In short, semantics is a definition of classes while structure is a representation of relationships (Tennis, 2011).

The researchers of the study first listed terminologies in the $K D C$, individually searched for the same or similar terminologies in the WebDewey website (http://dewey.org/webdewey) and compared the classifica- 
tion numbers to understand their hierarchies. The researchers compared the results and discussed any different interpretations found until they reached an agreement.

\section{RESULTS}

The majority of terminologies in the $K D C$ 's 510 to 519 on the subject of medical science and the $D D C$ 's 610 to 619 on the subject of medicine and health were matched but several unique subclasses or hierarchal placements were identified, representing the order of medical literature in Republic of Korea (South Korea) and the United States. Table 1 summarizes the overview of those identified differences with examples.

\begin{tabular}{|l|l|l|}
\hline & \multicolumn{1}{|c|}{ Prominent Case } & \multicolumn{1}{c|}{ Subtle Case } \\
\hline \multirow{5}{*}{ Terminology } & $\begin{array}{l}\text { New term/concept } \\
\text { inserted } \\
\text { e.g.) Oriental medi- } \\
\text { cine, Korean medicine } \\
\text { (KDC 519) }\end{array}$ & $\begin{array}{l}\text { Scopes (specificities) } \\
\text { e.g.) Nursing (KDC } \\
512.8)\end{array}$ \\
\hline \multirow{5}{*}{ Structure } & $\begin{array}{l}\text { Matched concepts but } \\
\text { differently ordered } \\
\text { e.g.) Clinical medicine } \\
\text { (KDC 512) }\end{array}$ & $\begin{array}{l}\text { Viewpoints (locational } \\
\text { differences) } \\
\text { e.g.) Nursing (KDC } \\
\text { 512.8) } \\
\text { e.g.) Emergency medi- } \\
\text { cine (KDC 512.6) }\end{array}$ \\
\hline
\end{tabular}

Table 1. Summary of major differences and examples

The observed cases appeared to be grouped into types: prominent influences and subtle influences. The prominent cases involve concepts that are unique to either one of the two cultures (Choi, 2018). Prominent influences are represented either by 1) terminological differences in establishing new subjects or 2) distinctive structural differences that manipulate the representation of concepts that the $D D C$ and the $K D C$ share. For example, the $K D C$ ' 519. Oriental medicine, Korean medicine' is an addition of a new subject. The $K D C$ '512. Clinical medicine' lists subjects corresponding to some of the $D D C$ 's numbers but these subject categories, including section number 512, demonstrate distinctive approaches to clinical medicine from the $D D C$. Subtle cases indicate differences in the structure or scope of a concept, which may reflect socio-cultural influences in two ways: 1) scopes or 2) viewpoints of a concept shared in both cultures (Choi, 2018).

The KDC '519. Oriental medicine, Korean medicine' demonstrate a prominent cultural difference in terminology that has no matching subjects in the $D D C$. The $D D C$ has a number for acupuncture as one of subordinated numbers to other therapies (615.89), but no class numbers dedicated to oriental medicine as a sub-discipline of medicine. Another prominent is '512. Clinical medicine' in the KDC. This section level number for clinical medicine is the result of an influence by the Universal Decimal Classification (UDC) system as the medical subjects were mostly based on the $U D C$ in the early development of the $K D C$ ( Oh, Bae, \& Yeo, 2002). On the other hand, the $D D C$ contains no numbers that exclusively address clinical medicine but notes clinical medicine directing users to either '616. Diseases,' or '616.075, Diagnosis and Prognosis.' Thus, in the DDC, those subordinated numbers to the $K D C 512$ are spread out to multiple places with different section numbers as shown in table 2 .

Several structural differences demonstrate prominent differences in concept structure as well. Concepts at higher levels in the hierarchy have more room for specific details on the topic, and the $K D C$ demonstrates this by having clinical medicine at the section level. To illustrate, the $K D C$ maintains a subject of '512. Clinical Medicine' at the section level, and consequently the subordinated subjects to clinical medicine are organized to address specific elements or aspects of clinical medicine under that number. For example, '512.8, Nursing' and '512.6, Emergency Medicine' are categorized as subordinated subjects to '512. Clinical medicine.' However, the $D D C$ places similar subject at the lower levels in the hierarchy of medical subjects, such as '610.73, Nursing and services of allied health personnel, 610.732-610.736, Nursing', and '616.025, Medical emergencies,' implying that they are facets of basic medical subjects. Notions of relative rank may exhibit a socio-cultural influence. The levels in hierarchy do not necessarily express ideas of superiority or inferiority, but merely implies that lower levels are part of those above them (Garnter, 2016). However, it is generally believed that the first concept listed on a higher level has more weight than those nested within the broader concepts one layer up (Gartner, 2016)

The scopes of the two systems differ particularly regarding the terminologies of captions and narrower categories for matched concepts in both systems. In the case of concepts that demonstrate different viewpoints, broader concepts or categories are not matched to the equivalent number of concepts even if many of their concepts are matched on lower levels. The new category of clinical medicine introduced not only structural differences but also differences in specificities. '(512.6) Emergency Medicine' in the KDC does not display any subordinated subjects, implying that no further specificities in the concept were made and, making the concept incomparable to topical specificities with the $D D C$. The differences in the scope and viewpoint of nursing are another example of such subtle cases. The $D D C$ exhibits detailed concepts of nursing without an inclusive broader category, while the $K D C$ presents a single category of ' 512.8 , Nursing' as one of subordinated subjects to clinical medicine. Some detailed categories for nursing types (e.g. private duty nursing, institutional nursing, public health nursing, and long-term care nursing) in the $D D C$ are generally matched with the $K D C$. The $K D C$, however, lists more diverse types of nursing by having an inclusive broader category of ' 512.8 , Nursing' at a higher level in the hierarchy. See table 2 for noticeable matches between medical subjects in the $K D C$ and $D D C$ that demonstrate differences in hierarchy, placement, and categories. 


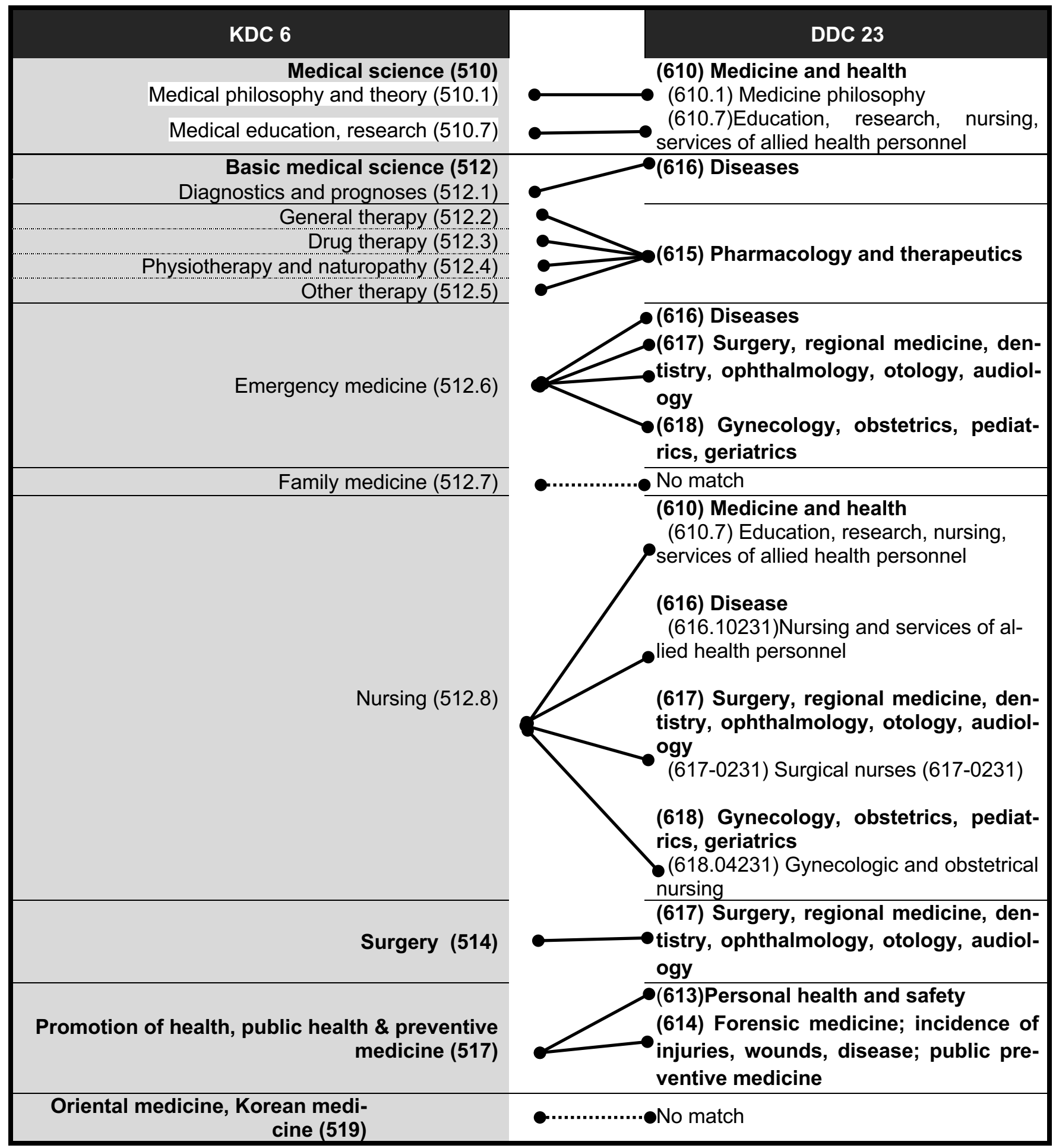

Table 1 Example mapping results between KDC and DD

\section{DISCUSSION AND CONCLUSION}

This preliminary study reports differences in medical science subject classification in the KDC and the DDC with the purpose of understanding the socio-cultural influences in two classificatory features: semantic content and structure. Despite the importance of standardization in medical science as well as similarities in the main purpose of the two systems, the comparison revealed the differences in both hierarchy and terminology. Some differences are more obvious while others are subtle. Our findings are aligned with the claim that these two essential constructs of classification, semantic contents and structures, are influenced by cultural and intellectual infrastructures (Olson, 2010). Terminological and structural differences in the medical subjects between $K D C$ and $D D C$ suggest that these two different classification systems were developed in distinct sociocultural contexts.

Organization, or the imposition of structure, is necessary to locate information resources (Norton, 2010). Information resources are positioned in a collection to impose structure with rules for placement and association (Rowley, 1992). The rules and criteria for organizing are based upon agreement on the intended use of information resources and 
the anticipated users (Norton, 2010). However, the organization of social knowledge and its impact on society through classification systems can be sensitive and critical issues with the use of health information organization systems can occur as consequence of classification, which may lead to other issues such as political and ethical problems. For example, different cultures have different ways of defining the moment of birth. That difference causes conflict in creating a definition in the International Classification of Disease (ICD). the ICD reflects the charged political and ethical atmosphere surrounding controversial topics, forcing some definitions to be abandoned or silenced and others to appear exotic or overly convoluted (Bowker \& Star, 2000).

The case of the $K D C$ 's cross-cultural adaptation of the $D D C$ in medical subjects demonstrates how KOSs carry sociocultural influences. The observed differences support current understanding of the subject in different socio-cultural contexts. The $K D C$ 's adaptation of the $D D C$ is a case of a national library classification system adopting the dominant system, making it locally useful within the influences of western scientific disciplines. At the same time, the $K D C$ has been developed for its own needs to serve the public libraries in the Republic of Korea (South Korea). In the early development of the $K D C$, many parts of main classes and class numbers at the division level were borrowed from the $D D C$, while section levels and lower levels were developed for Korean needs. To understand this as a result of the $K D C$ s adoption of the $D D C$, a comparison of class numbers at the lower levels is desired. These inspections would reveal developmental histories of the two systems as well as sociocultural impacts on the classification systems, and would enhance interoperability among different classification systems.

This study of classifications systems in a cross-cultural comparison would serve as a comparative domain analysis of library systems organizing medicine literature. As both the $K D C$ and the $D D C$ systems, are widely used in public libraries, the organization of medical literature for use of classification systems could inhibit or assist public library user access to the resources. Although the distinct developments of both systems in their socio-cultural contexts have been empirically examined demonstrating plural cultural views, standardization of medical subjects could enhance user accessibility of medical resources. For future studies, we plan to investigate widely used medical subject headings and classification systems (e.g., MeSH) as well as standardized classification systems in various medical fields (e.g., International Classification of Diseases (ICD)) to better understand the differences and ultimately improve the interoperability of related systems.

\section{REFERENCES}

Armitage, G. (1999). Development of a classification system for periodontal diseases and conditions. Annals of periodontology, 4(1), 1-6.

Ballard, J. (1921). Medical Classification. Bulletin of the Medical Library Association 11(1-4), 13-18.

Bowker, G. C., \& Star, S. L. (2000). Sorting things out: Classification and its consequences: MIT press.

Choi, I. (2018). Toward a Model of Intercultural Warrant: A Case of the Korean Decimal Classification's Crosscultural Adaptation of the Dewey Decimal Classification. The University of Wisconsin-Milwaukee,

Chute, C. G. (2000). Clinical classification and terminology: some history and current observations. Journal of the American Medical Informatics Association 7(3), 298-303.

Gartner, R. (2016). Metadata : shaping knowledge from antiquity to the semantic web. Springer.

Hantrais, L. (2008). International comparative research: Theory, methods and practice: Macmillan International Higher Education.

Norton, M. J. (2010). Introductory concepts in information science (2nd ed.). Medford, N.J.: Published on behalf of the American Society for Information Science and Technology by Information Today.

Oh, D. G. Bae., Y.; Yeo, J. (2002). Understanding of KDC. Seoul, South Korea: Taeil Publishing.

Olson, H. (2010). Social influences on classification. Paper presented at the Encyclopedia of library and information sciences.

Rowley, J. (1992). Organizing knowledge: an introduction to information retrieval: Gower.

Tennis, J. T. (2011). Is There a New Bibliography? Cataloging \& Classification Quarterly 49(2), 121-126.

U.S. National Library of Medicine. (2018). Cross-Cultural Comparison. In $\mathrm{MeSH}$. 